Review Article

\title{
Leray-Schauder Fixed Point Theorems for Block Operator Matrix with an Application
}

\author{
Mohamed Amine Farid (D), El Miloudi Marhrani $(\mathbb{D}$, and Mohamed Aamri (D) \\ L3A Laboratory, Department of Mathematics and Computer Sciences, Faculty of Sciences Ben M'sik, University of Hassan II, \\ Casablanca, Morocco \\ Correspondence should be addressed to Mohamed Amine Farid; amine.farid17@gmail.com
}

Received 10 March 2021; Revised 16 June 2021; Accepted 10 July 2021; Published 27 July 2021

Academic Editor: Huseyin Isik

Copyright $(92021$ Mohamed Amine Farid et al. This is an open access article distributed under the Creative Commons Attribution License, which permits unrestricted use, distribution, and reproduction in any medium, provided the original work is properly cited.

In this paper, we establish some new variants of Leray-Schauder-type fixed point theorems for a $2 \times 2$ block operator matrix defined on nonempty, closed, and convex subsets $\Omega$ of Banach spaces. Note here that $\Omega$ need not be bounded. These results are formulated in terms of weak sequential continuity and the technique of De Blasi measure of weak noncompactness on countably subsets. We will also prove the existence of solutions for a coupled system of nonlinear equations with an example.

\section{Introduction}

With the development of new problems in diverse fields of sciences as well as in physical, biological, and social sciences, the theory of fixed point and its applications are very diverse and continuously growing. Also, the theory of block operator matrix is a subject of great interest thanks to the useful applications for studying some systems of integral equations as well as systems of partial or ordinary differential equations. Recent work has employed the fixed point technique for the operator matrix with nonlinear entries acting on Banach spaces or Banach algebras for studying the existence of solutions for several classes of systems of nonlinear integral equations, see, for example, [1-5]. These operators are defined by a $2 \times 2$ block operator matrix:

$$
\left(\begin{array}{ll}
A & B \\
C & D
\end{array}\right)
$$

Based on new generalized Schauder and Krasnoselskii fixed point theorems for the block operator matrix (1), Ben Amar et al., in [6], have established some results for a coupled system of differential equations on $L_{p} \times L_{p}$ for $p \in(1, \infty)$, under abstract boundary conditions of Rotenberg's model type. These last equations were proposed by M. Rotenberg and model the evolution of a cell population
[7]. Due to the lack of compactness on $L_{1}$ spaces, the study in [6] did not cover the case $p=1$. Later, Jeribi et al. [1] proposed to extend the results of Amar et al. [6] to the case $p=1$ by establishing new variants of fixed point theorems for (1), and their analysis was carried out via arguments of weak topology and, particularly, the technique of measures of weak noncompactness. In the above quoted works, the assumptions that $I-A$ or $I-D$ are invertible play a fundamental role in the arguments. Jeribi et al., in [8], were interested in studying the case when $I-A$ is not injective and established some fixed point theorems for operator (1), involving multivalued maps acting on Banach spaces. This way, their results were formulated in terms of weak sequential continuity and the technique of De Blasi measure of weak noncompactness. The results obtained are then applied to the two-dimensional nonlinear functional integral equation:

$$
\left\{\begin{array}{l}
x(t)=k(t, x(t))+\left[q(t)+\int_{0}^{\sigma(t)} g(t, s, y(s)) \mathrm{d} s\right] \cdot u, \\
y(t)=\phi\left(t, \int_{0}^{t} \frac{t}{t+s} w(s, x(s)) \mathrm{d} s\right) \cdot v+a(t) y(t),
\end{array}\right.
$$


for all $t \in J=[0,1]$, where $u, v \in X$ with $u \neq 0$ and $v \neq 0$; here, $X$ is a Banach space. And, $\sigma: J \longrightarrow J$, $g: J \times J \times X \longrightarrow X, k, w: J \times J \longrightarrow \mathbb{R}$, and $b: J \longrightarrow X$ are suitably defined functions.

The main purpose of this paper is to obtain some new variants of Leray-Schauder-type fixed point theorem for operator (1) on a Banach space. From application, we discuss the existence of solutions to problem (2) in a suitable Banach space and an example of a nonlinear integral equation in the Banach space $\mathscr{C}([0,1], \mathbb{R})$.

Note that system (2) can be written as a fixed point problem:

$$
\left(\begin{array}{l}
x(t) \\
y(t)
\end{array}\right)=\left(\begin{array}{cc}
k(t, \cdot) & G(t, \cdot) \\
\varphi(t, \cdot) & a(t)
\end{array}\right)\left(\begin{array}{c}
x(t) \\
y(t)
\end{array}\right)
$$

where

$$
\left\{\begin{array}{l}
G(t, y(t))=\left[q(t)+\int_{0}^{\sigma(t)} g(t, s, y(s)) \mathrm{d} s\right] \cdot u \\
\varphi(t, x(t))=\phi\left(t, \int_{0}^{t} \frac{t}{t+s} w(s, x(s)) \mathrm{d} s\right) \cdot v .
\end{array}\right.
$$

The present paper is built up as follows. In Section 2, we introduce the necessary definitions and preliminary concepts. Section 3 is devoted to present some new variants of Leray-Schauder-type fixed point theorems for a $2 \times 2$ block operator matrix maps acting on Banach spaces. Finally, in Section 4, we apply Corollary 1 in order to discuss the existence of solutions for problem (2).

\section{Basic Definitions and Preliminary Concepts}

In this section, we give some essential definitions, properties, and theorems of fixed point theory, which should be used in the present paper. Throughout this paper, unless otherwise mentioned, $X$ denotes a Banach space endowed with the norm $\|\cdot\|$ and with the zero element $\theta, B_{r}$ denotes the closed ball in $X$ centered at $\theta$ with radius $r>0$, and $P_{b d}(X)$ denotes the collection of all nonempty bounded subsets of $X$. Moreover, we write $x_{n} \longrightarrow x$ and $x_{n} \rightarrow x$ to denote, respectively, the strong convergence and the weak convergence of a sequence $\left\{x_{n}\right\}_{n}$ to $x$. We say that a map $T: X \longrightarrow X$ is weakly sequentially continuous if, for every sequence $\left\{x_{n}\right\}_{n} \subset X$ with $x_{n} \rightarrow x$, we have $T x_{n} \rightarrow T x$.

The De Blasi measure of weak noncompactness [9] is the map $\omega: P_{b d}(X) \longrightarrow[0, \infty)$ defined by
The De Blasi measure of weak noncompactness satisfies the following properties. For a proof, we refer the reader to $[9,10]$.

Lemma 1. Let $M_{1}$ and $M_{2}$ be in $P_{b d}(X)$, and we have

(i) If $M_{1} \subset M_{2}$, then $\omega\left(M_{1}\right) \leq \omega\left(M_{2}\right)$

(ii) $\omega\left(M_{1}\right)=0$ if and only if $M_{1}$ is relatively weakly compact

(iii) $\omega\left(\overline{M_{1}^{w}}\right)=\omega\left(M_{1}\right)$, where $\overline{M_{1}^{w}}$ is the weak closure of $M_{1}$

(iv) $\omega\left(\lambda M_{1}\right)=|\lambda| \omega\left(M_{1}\right)$, for all $\lambda \in \mathbb{R}$

(v) $\omega\left(\overline{c o}\left(M_{1}\right)\right)=\omega\left(M_{1}\right)$, where $\overline{c o}\left(M_{1}\right)$ is the closed convex hull of $M_{1}$

(vi) $\omega\left(M_{1}+M_{2}\right) \leq \omega\left(M_{1}\right)+\omega\left(M_{2}\right)$

(vii) $\omega\left(M_{1} \cup\{x\}\right)=\omega\left(M_{1}\right)$, for all $x \in X$

In [10], Appell and De Pascale proved that, in $L^{1}$-spaces, the maps $\omega(\cdot)$ has the following form:

$$
\omega(M)=\limsup _{\varepsilon \longrightarrow 0}\left\{\sup _{\psi \in M}\left[\int_{D}\|\psi(t)\|_{X} \mathrm{~d} t: \operatorname{meas}(D) \leq \varepsilon\right]\right\} .
$$

For all bounded subsets $M$ of $L^{1}(\Omega, X)$, where $X$ is a finite dimensional Banach space and meas $(\cdot)$ denotes the Lebesgue measure, we recall the following definitions.
Definition 1. Let $\Omega$ be a subset of a Banach space $X$, and $k \in[0,1)$. Let $T: \Omega \longrightarrow X$ be a mapping, we say that

1. $T$ is $k$-contractive if $\omega(T(M)) \leq k \omega(M)$ for any bounded set $M \subset \Omega$

(2) $T$ is condensing if $\omega(T(M))<\omega(M)$ for any bounded set $M \subset \Omega$ with $\omega(M)>0$

(3) $T$ is countably $k$-contractive if $\omega(T(M)) \leq k \omega(M)$ for any countable bounded set $M \subset \Omega$

(4) $T$ is countably condensing if $\omega(T(M))<\omega(M)$ for any countable bounded set $M \subset \Omega$ with $\omega(M)>0$

Clearly, every $k$-contractive is countably $k$-contractive. Now, $T$ is said to be $k$-Lipschitzian if $\|T x-T y\| \leq k\|x-y\|$ with $k \in[0, \infty)$. If $k \in[0,1), T$ is called a contraction.

Definition 2. A mapping $T: X \longrightarrow X$ is said to be weakly compact if $T(M)$ is relatively weakly compact for every nonempty bounded subset $M \subseteq X$.

Following [11], we recall the next definition.

Definition 3. A mapping $T: X \longrightarrow X$ is called to be a separate contraction if there exist two functions $\varphi, \psi:[0, \infty) \longrightarrow[0, \infty)$, satisfying

(1) $\psi$ is strictly increasing and $\psi(0)=0$

(2) $\|T x-T y\| \leq \varphi(\|x-y\|)$ for all $x, y \in X$

(3) $\psi(r)+\varphi(r) \leq r$ for any $r>0$ 
Note that every contraction mapping is a separate contraction mapping.

Definition 4 (see [12]). A mapping $T: X \longrightarrow X$ is a nonlinear contraction mapping if there exists a continuous nondecreasing function $\varphi:[0, \infty) \longrightarrow[0, \infty)$ satisfying

(1) $\varphi(r)<r$ for any $r>0$

(2) $\|T x-T y\| \leq \varphi(\|x-y\|)$ for all $x, y \in X$

Definition 5. An operator $T: X \longrightarrow X$ is said to be $\psi$-expansive if there exists a function $\psi:[0, \infty) \longrightarrow[0, \infty)$ such that

(1) $\psi(0)=0$

(2) $\psi(r)>r$, for any $r>0$

(3) $\psi$ is either continuous or nondecreasing

(4) $\|T x-T y\| \geq \psi(\|x-y\|)$ for all $x, y \in X$

In particular, for $\psi(t)=h t$ with $h>1$, then $T$ is an expansive mapping (see [13]). The following results are the nonlinear alternatives of Leray-Schauder-type states in [14].

Theorem 1. Let $\Omega$ be a nonempty closed and convex subset of a Banach space $X$ and $U \subset \Omega$ be a weakly open subset of $\Omega$ with $\theta \in U$ such that $\overline{U^{w}}$ is a weakly compact subset of $\Omega$ and $F: \overline{U^{w}} \longrightarrow \Omega$ is a weakly sequentially continuous mapping.

Then, either

(A1) F has a fixed point or

(A2) there is a point $x \in \partial_{\Omega} U$ and $\lambda \in(0,1)$ with $x=\lambda F x$, where $\partial_{\Omega} U$ denotes the weak boundary of $U$ in $\Omega$.

Remark 1. In Theorem 1, the condition " $\overline{U^{w}}$ is a weakly compact" can be replaced by " $F\left(\overline{U^{w}}\right)$ is relatively weakly compact," for the proof, see Remark 3.2 in [14].

Theorem 2. Let $\Omega$ be a nonempty closed and convex subset of a Banach space $X$ and $U \subset \Omega$ be a weakly open subset of $\Omega$ with $\theta \in U$. Assume that $F: \overline{U^{w}} \longrightarrow \Omega$ is a weakly sequentially continuous and condensing map with $F\left(\overline{U^{w}}\right)$ bounded. Then, either

(A1) F has a fixed point or

(A2) there is a point $x \in \partial_{\Omega} U$ and $\lambda \in(0,1)$ with $x=\lambda F x$, where $\partial_{\Omega} U$ denotes the weak boundary of $U$ in $\Omega$.

Amar et al., in [15], showed that the condition "condensing" in Theorem 2 can be relaxed by the assumption "countably condensing."

Theorem 3. Let $\Omega$ be a nonempty closed and convex subset of a Banach space $X$ and $U \subset \Omega$ be a weakly open subset of $\Omega$ with $\theta \in U$. Assume that $F: \overline{U^{w}} \longrightarrow \Omega$ is a weakly sequentially continuous and countably condensing map with $F\left(\overline{U^{w}}\right)$ bounded. Then, either
(A1) F has a fixed point or

(A2) there is a point $x \in \partial_{\Omega} U$ and $\lambda \in(0,1)$ with $x=\lambda F x$, where $\partial_{\Omega} U$ denotes the weak boundary of $U$ in $\Omega$

The following results are crucial for our purposes.

Lemma 2 (see [16]). Let $\Omega$ be a subset of a Banach space $X$ and let $T: \Omega \longrightarrow X$ be a $k$-Lipschitzian map. Assume that $T$ is a sequentially weakly continuous map. Then, $\omega(T(M)) \leq k \omega(M)$ for each bounded subset $M$ of $\Omega$; here, $\omega(\cdot)$ stands for the De Blasi measure of weak noncompactness.

Lemma 3 (see [17]). Let $K$ be a Hausdorff compact space and $X$ be a Banach space. A bounded sequence $\left\{f_{n}\right\}_{n} \subset \mathscr{C}(K, X)$ converges weakly to $f \in \mathscr{C}(K, X)$ if and only if, for every $t \in K$, the sequence $\left\{f_{n}(t)\right\}_{n}$ converges weakly (in $\left.X\right)$ to $f(t)$.

\section{Main Result}

In this section, we give some new variants of Leray-Schauder for the operator (1). The first result is formulated as follows.

Theorem 4. Let $\Omega$ be a nonempty closed and convex subset of a Banach space $X$ and $U \subset \Omega$ be a weakly open subset of $\Omega$ with $\theta \in U$. Let $A, C: \overline{U^{w}} \longrightarrow X$ and $B, D: X \longrightarrow X$ be four operators such that

(i) $A$ is linear and bounded, and there is $p \in \mathbb{N}^{*}$ such that $A^{p}$ is a separate contraction

(ii) $B$ and $C$ are weakly sequentially continuous and $C\left(\overline{U^{w}}\right)$ is relatively weakly compact

(iii) $D$ is linear and bounded, and there is $p \in \mathbb{N}^{*}$ such that $D^{p}$ is a separate contraction

(iv) $(I-A)^{-1} B(I-D)^{-1} C x \in \Omega$, for all $x \in \overline{U^{w}}$

Then, either

(A1) the block operator matrix (1) has a fixed point or

(A2) there exists $(u, v) \in \partial_{\Omega} U \times X$ and $\lambda \in(0,1)$ such that

$$
\left(\begin{array}{cc}
A & \lambda B \\
C & D
\end{array}\right)\left(\begin{array}{l}
u \\
v
\end{array}\right)=\left(\begin{array}{l}
u \\
v
\end{array}\right),
$$

where $\partial_{\Omega} U$ denotes the weak boundary of $U$ in $\Omega$.

Proof. If we refer to Lemma 1.2 in [11] and to page 39 in [18], we can prove that $(I-A)^{-1}$ and $(I-D)^{-1}$ exist and are weakly continuous. Hence, we can define the mapping $F: \overline{U^{w}} \longrightarrow \Omega$ by

$$
F x=(I-A)^{-1} B(I-D)^{-1} C x .
$$

In view of Theorem 1, it suffices to establish that $F$ is weakly sequentially continuous and $F\left(\bar{U}^{w}\right)$ is relatively weakly compact.

We have $(I-A)^{-1}$ and $(I-D)^{-1}$, and $B$ and $C$ are weakly sequentially continuous; then, $F$ is weakly 
sequentially continuous, and because $C\left(\overline{U^{w}}\right)$ is relatively weakly compact, we get $F\left(\overline{U^{w}}\right)$ is relatively weakly compact. Hence, either

(1) $F$ has a fixed point or

(2) there is a point $u \in \partial_{\Omega} U$ and $\lambda \in(0,1)$ with $u=\lambda F u$.

In the first case, the vector $y=(I-D)^{-1} C x$ solves the problem, whereas in the second case we use the vector $v=$ $(I-D)^{-1} \mathrm{Cu}$ to achieve the proof.

\section{Remark 2}

(1) Theorem 4 remains true if we suppose that $A^{p}$ or $D^{p}$ is a nonlinear contraction

(2) Theorem 4 is a generalization of Theorem 4 and Theorem 3.2 in [19]

We also have the following result.

Theorem 5. Let $\Omega$ be a nonempty closed and convex subset of a Banach space $X$ and $U \subset \Omega$ be a weakly open subset of $\Omega$ with $\theta \in U$. Let $A, C: \overline{U^{w}} \longrightarrow X$ and $B, D: X \longrightarrow X$ be four operators such that

(i) $A\left(\overline{U^{w}}\right)$ and $C\left(\overline{U^{w}}\right)$ are relatively weakly compact

(ii) $A, B$, and $C$ are weakly sequentially continuous

(iii) $D$ is linear and bounded, and there is $p \in \mathbb{N}^{*}$ such that $D^{p}$ is a separate contraction

(iv) $A x+B(I-D)^{-1} C x \in \Omega$, for all $x \in \overline{U^{w}}$

Then, either

(A1) the block operator matrix (1) has a fixed point or

(A2) there exists $(u, v) \in \partial_{\Omega} U \times X$ and $\lambda \in(0,1)$ such that

$$
\left(\begin{array}{cc}
\lambda A & \lambda B \\
C & D
\end{array}\right)\left(\begin{array}{l}
u \\
v
\end{array}\right)=\left(\begin{array}{l}
u \\
v
\end{array}\right),
$$

where $\partial_{\Omega} U$ denotes the weak boundary of $U$ in $\Omega$.

Proof. The reasoning in the proof of Theorem 4 yields that $(I-D)^{-1}$ exists and is weakly continuous. Hence, we can define the mapping $F: \overline{U^{w}} \longrightarrow \Omega$ by

$$
F x=A x+B(I-D)^{-1} C x .
$$

We can see that $F$ is weakly sequentially continuous because $A, B, C$, and $(I-D)^{-1}$ are weakly sequentially continuous. The assumption (i) proves that $F\left(\overline{U^{w}}\right)$ is relatively weakly compact. Hence, by Theorem 1, we have

(1) $F$ has a fixed point or

(2) There is a point $u \in \partial_{\Omega} U$ and $\lambda \in(0,1)$ with $u=\lambda F u$

In the first case, the vector $y=(I-D)^{-1} x$ solves the problem, whereas in the second case we use the vector $v=$ $(I-D)^{-1} \mathrm{Cu}$ to achieve the proof.
Remark 3

(1) In Theorem 5, we can replace the assumption " $A x+B(I-D)^{-1} C x \in \Omega$, for all $x \in \overline{U^{w}}$," by “ $x=A x+B(I-D)^{-1} C y \in \Omega$, for all $y \in \overline{U^{w}}$ implies $x \in \Omega$ "

(2) Theorem 5 is a generalization of Theorem 3.3 in [19]

Thus, we give the following result

Theorem 6. Let $\Omega$ be a nonempty closed and convex subset of a Banach space $X$ and $U \subset \Omega$ be a weakly open subset of $\Omega$ with $\theta \in U$. Let $A, C: \overline{U^{w}} \longrightarrow X$ and $B, D: X \longrightarrow X$ be four operators such that

(i) $A\left(\overline{U^{w}}\right)$ and $C\left(\overline{U^{w}}\right)$ are relatively weakly compact

(ii) $A, B$, and $C$ are weakly sequentially continuous

(iii) $D$ is linear and bounded, and there is $p \in \mathbb{N}^{*}$ such that $D^{P}$ is a separate contraction

(iv) $A x+B(I-D)^{-1} C x \in \Omega$, for all $x \in \overline{U^{w}}$

Then, either

(A1) the block operator matrix (1) has a fixed point or

(A2) there exists $(u, v) \in \partial_{\Omega} U \times X$ and $\lambda \in(0,1)$ such that

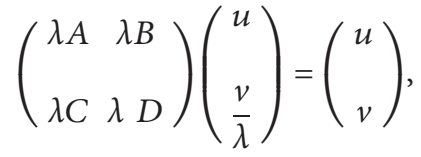

where $\partial_{\Omega} U$ denotes the weak boundary of $U$ in $\Omega$.

Proof. Reasoning as in the proof of Theorem 5, we obtain

(1) $F$ has a fixed point or

(2) There is a point $u \in \partial_{\Omega} U$ and $\lambda \in(0,1)$ with $u=\lambda F u$

In the first case, the vector $y=(I-D)^{-1} x$ solves the problem, whereas in the second case, we use the vector $v=$ $\lambda(I-D)^{-1} \mathrm{Cu}$ to achieve the proof.

Remark 4. Theorem 6 is a generalization of Theorem 3.4 in [19].

Theorem 7. Let $\Omega$ be a nonempty closed and convex subset of Banach space $X$ and $U \subset \Omega$ be a weakly open subset of $\Omega$ with $\theta \in U$. Let $A, C: \overline{U^{w}} \longrightarrow X$ and $B, D: X \longrightarrow X$ be four weakly sequentially continuous operators such that

(i) $A\left(\overline{U^{w}}\right)$ and $C\left(\overline{U^{w}}\right)$ are relatively weakly compact

(ii) $D$ is a contraction with $C\left(\overline{U^{w}}\right) \subset(I-D)(\Omega)$

(iii) $A x+B(I-D)^{-1} C x \in \Omega$, for all $x \in \overline{U^{w}}$

Then, either

(A1) the block operator matrix (1) has a fixed point or

(A2) there exists $(u, v) \in \partial_{\Omega} U \times X$ and $\lambda \in(0,1)$ such that 


$$
\left(\begin{array}{cc}
\lambda A & \lambda B \\
C & D
\end{array}\right)\left(\begin{array}{l}
u \\
v
\end{array}\right)=\left(\begin{array}{l}
u \\
v
\end{array}\right)
$$

where $\partial_{\Omega} U$ denotes the weak boundary of $U$ in $\Omega$.

Proof. By assumption (ii), we can see that $(I-D)^{-1}$ exists and continuous; hence, from Theorem 5 , we deduce the desired result.

Inspired by [20], we deduce the following result.

Corollary 1. Let $\Omega$ be a nonempty closed and convex subset of a Banach space $X$ and $U \subset \Omega$ be a weakly open subset of $\Omega$ with $\theta \in U$. Let $A, C: \overline{U^{w}} \longrightarrow X$ and $B, D: X \longrightarrow X$ be four weakly sequentially continuous operators such that

(i) $A\left(\overline{U^{w}}\right)$ and $C\left(\overline{U^{w}}\right)$ are relatively weakly compact

(ii) $D$ is a contraction with $C\left(\overline{U^{w}}\right) \subset(I-D)(\Omega)$

(iii) $A x+B(I-D)^{-1} C x \in \Omega$ for all $x \in \overline{U^{w}}$

In addition, assume that

$$
\lambda A u+\lambda B v \neq u,
$$

for all $(u, v) \in \partial_{\Omega} U \times X$ and $\lambda \in(0,1)$. Then, the set of fixed points of the block operator matrix (1) in $\overline{U^{w}} \times X$ is nonempty.

In the next results, we explore the case where $A$ and $D$ are nonlinear.

Theorem 8. Let $\Omega$ be a nonempty closed and convex subset of a Banach space $X$ and $U \subset \Omega$ be a weakly open subset of $\Omega$ with $\theta \in U$. Let $A, C: \overline{U^{w}} \longrightarrow X$ and $B, D: X \longrightarrow X$ be four weakly sequentially continuous operators such that (i) $(I-D)^{-1}$ exists on $C(\Omega)$

(ii) $A\left(\overline{U^{w}}\right)$ is relatively weakly compact and $B$ is countably $\beta$-contractive

(iii) $C$ is countably $\gamma$-contractive and $D$ is countably $\delta$-contractive with $\gamma+\delta<1$

(iv) $\left(A+B(I-D)^{-1} C\right)\left(\overline{U^{w}}\right)$ is a bounded subset of $\Omega$

Then, either

(A1) the block operator matrix (1) has a fixed point or

(A2) there exists $(u, v) \in \partial_{\Omega} U \times X$ and $\lambda \in(0,1)$ such that

$$
\left(\begin{array}{cc}
\lambda A & \lambda B \\
C & D
\end{array}\right)\left(\begin{array}{l}
u \\
v
\end{array}\right)=\left(\begin{array}{l}
u \\
v
\end{array}\right)
$$

where $\partial_{\Omega} U$ denotes the weak boundary of $U$ in $\Omega$.

Proof. We defend the operator $F: \overline{U^{w}} \longrightarrow \Omega$ by

$$
F x=A x+B(I-D)^{-1} C x .
$$

We show that $F$ is weakly sequentially continuous and countably condensing.

Let $\left\{x_{n}\right\}_{n}$ be a sequence in $\Omega$ such that $x_{n} \rightarrow x$; since $C$ is weakly sequentially continuous, then the set $\left\{C x_{n}: n \in \mathbb{N}\right\}$ is relatively weakly compact. Note that

$$
(I-D)^{-1} C=C+D(I-D)^{-1} C \text {. }
$$

We have $D$ is countably $\delta$-contractive; then,

$$
\begin{aligned}
\omega\left(\left\{(I-D)^{-1} C x_{n}: n \in \mathbb{N}\right\}\right) & \leq \omega\left(\left\{C x_{n}: n \in \mathbb{N}\right\}\right)+\omega\left(\left\{D(I-D)^{-1} C x_{n}: n \in \mathbb{N}\right\}\right) \\
& \leq \omega\left(\left\{D(I-D)^{-1} C x_{n}: n \in \mathbb{N}\right\}\right) \\
& \leq \delta \omega\left(\left\{(I-D)^{-1} C x_{n}: n \in \mathbb{N}\right\}\right) \\
& <\omega\left(\left\{(I-D)^{-1} C x_{n}: n \in \mathbb{N}\right\}\right),
\end{aligned}
$$

which implies that $\omega\left(\left\{(I-D)^{-1} C x_{n}: n \in \mathbb{N}\right\}\right)=0$; then, $\left\{(I-D)^{-1} C x_{n}: n \in \mathbb{N}\right\}$ is relatively weakly compact. Consequently, there exists a subsequence $\left\{x_{\sigma(n)}\right\}_{n \in \mathbb{N}}$ of $\left\{x_{n}\right\}_{n \in \mathbb{N}}$ such that $(I-D)^{-1} C x_{\sigma(n)} \rightarrow y$. From $(16)$ and because $C$ and $D$ are weakly sequentially continuous, we get $C x+D y=y$; then, $(I-D)^{-1} C x=y$; consequently, $(I-D)^{-1} C x_{\sigma(n)} \rightarrow(I-D)^{-1} C x$. Now, we show that $(I-D)^{-1} C x_{n} \rightarrow(I-D)^{-1} C X$. Suppose the contrary; then, there exists a subsequence $\left\{x_{\varphi(n)}\right\}_{n \in \mathbb{N}}$ of $\left\{x_{n}\right\}_{n \in \mathbb{N}}$ and a weak neighborhood $V$ of $(I-D)^{-1} C x$ such that $(I-D)^{-1} C x_{\varphi(n)} \notin V$ for all $n \in \mathbb{N}$. Since $x_{\varphi(n)} \rightarrow x$, then arguing as before, we may extract a subsequence $\left\{x_{\phi(n)}\right\}_{n \in \mathbb{N}}$ of $\left\{x_{\varphi(n)}\right\}_{n \in \mathbb{N}}$ such that $(I-D)^{-1} C x_{\phi(n)} \rightarrow(I-D)^{-1} C x$, which is a contraction. As a result, $(I-D)^{-1} C$ is weakly sequentially continuous. Because $A$ and $B$ are weakly sequentially continuous, we get $A+B(I-D)^{-1} C$ is weakly sequentially continuous.

Now, we show that $A+B(I-D)^{-1} C$ is countably condensing. Let $M$ be a countably subset of $\overline{U^{w}}$ such that $\omega(M)>0$; by (16), we have

$$
\begin{aligned}
\omega\left((I-D)^{-1} C(M)\right) & \leq \omega(C(M))+\omega\left(D(I-D)^{-1} C(M)\right) \\
& \leq \gamma \omega(M)+\delta \omega\left((I-D)^{-1} C(M)\right) .
\end{aligned}
$$

Then,

$$
\omega\left((I-D)^{-1} C(M)\right) \leq \frac{\gamma}{1-\delta} \omega(M) \leq \omega(M) .
$$

Using the subadditivity of the De Blasi measure of weak noncompactness, we obtain 


$$
\begin{aligned}
\omega(F(M)) & \leq \omega(A(M))+\omega\left(B(I-D)^{-1} C(M)\right) \\
& \leq \beta \omega(M) \\
& <\omega(M) .
\end{aligned}
$$

Hence, $F$ is countably condensing.

Then, by Theorem 3, we obtain

(1) $F$ has a fixed point or

(2) There is a point $u \in \partial_{\Omega} U$ and $\lambda \in(0,1)$ with $u=\lambda F u$

In the first case, the vector $y=(I-D)^{-1} C x$ solves the problem, whereas in the second case, we use the vector $v=$ $(I-D)^{-1} \mathrm{Cu}$ to achieve the proof.

Remark 5. In Theorem 8, we can replace the De Blasi measure of weak noncompactness $\omega$ by any subadditive measures of weak noncompactness on $X$.

In Theorem 8 , condition (i) is difficult to verify; in the following, we will change it under weaker conditions.

Theorem 9. Let $\Omega$ be a nonempty closed and convex subset of a Banach space $X$ and $U \subset \Omega$ be a weakly open subset of $\Omega$ with $\theta \in U$. Let $A, C: \overline{U^{w}} \longrightarrow X$ and $B, D: X \longrightarrow X$ be four weakly sequentially continuous operators such that

(i) $(I-D)$ is $\psi$-expansive and $C(\Omega) \subset(I-D)(X)$

(ii) $A\left(\overline{U^{w}}\right)$ is relatively weakly compact and $B$ is countably $\beta$-contractive

(iii) $C$ is countably $\gamma$-contractive and $D$ is countably $\delta$-contractive with $\gamma+\delta<1$

(iv) $\left(A+B(I-D)^{-1} C\right)\left(\overline{U^{w}}\right)$ is a bounded subset of $\Omega$

Then, either

(A1) the block operator matrix (1) has a fixed point or

(A2) there exists $(u, v) \in \partial_{\Omega} U \times X$ and $\lambda \in(0,1)$ such that

$$
\left(\begin{array}{cc}
\lambda A & \lambda B \\
C & D
\end{array}\right)\left(\begin{array}{l}
u \\
v
\end{array}\right)=\left(\begin{array}{l}
u \\
v
\end{array}\right),
$$

where $\partial_{\Omega} U$ denotes the weak boundary of $U$ in $\Omega$.

Proof. Let $x, y \in X$ such that $x \neq y$; since $(I-D)$ is $\psi$-expansive, we obtain

$$
\|(I-D) x-(I-D) y\| \geq \psi(\|x-y\|)>0,
$$

Thus, $(I-D): X \longrightarrow(I-D)(X)$ is bijective, and because $C(\Omega) \subset(I-D)(X)$, we get $(I-D)^{-1}$ is well defined on $C(\Omega)$. Now, reasoning as in the proof of Theorem 8 , we get the desired result.

Theorem 10. Let $\Omega$ be a nonempty closed and convex subset of a Banach space $X$ and $U \subset \Omega$ be a weakly open subset of $\Omega$ with $\theta \in U$. Let $A, C: \overline{U^{w}} \longrightarrow X$ and $B, D: X \longrightarrow X$ be four weakly sequentially continuous operators such that (i) $B$ is countably $\beta$-contractive and $A\left(\overline{U^{w}}\right)$ is relatively weakly compact

(ii) $T_{y}^{p}$ is expansive for some $p \in \mathbb{N}$ and each $y \in X$, where $T_{y} x=D x+y$ for $x \in X$

(iii) $D$ is a contraction with constant $0<\delta<1$ and $C$ is countably $\gamma$-contractive with $\gamma+\delta<1$

(iv) $\left(A+B(I-D)^{-1} C\right)\left(\overline{U^{w}}\right)$ is a bounded subset of $\Omega$

Then, either

(A1) the block operator matrix (1) has a fixed point, or

(A2) there exists $(u, v) \in \partial_{\Omega} U \times X$ and $\lambda \in(0,1)$ such that

$$
\left(\begin{array}{cc}
\lambda A & \lambda B \\
C & D
\end{array}\right)\left(\begin{array}{l}
u \\
v
\end{array}\right)=\left(\begin{array}{l}
u \\
v
\end{array}\right),
$$

where $\partial_{\Omega} U$ denotes the weak boundary of $U$ in $\Omega$.

Proof. By assumptions (i) and (ii) with Lemma 2.3 in [21], we get $(I-D)^{-1} C$ is well defend. And, by assumption (i) and Lemma 2 , we have that $D$ is countably $\delta$-contractive. Now, reasoning as in the proof of Theorem 8 , we get the desired result.

Remark 6. Theorem 10 remains true if we suppose that $T_{y}^{p}$ is contractive.

\section{Application}

Let $X$ be a Banach space. As usual, we will denote by $E=$ $\mathscr{C}(J, X)$ the Banach space of all $X$-valued continuous functions defined on $J=[0,1]$. We equip the space $E$ with its standard norm:

$$
\|x\|_{\infty}=\sup _{t \in J}\|x(t)\| .
$$

The goal of this section is to apply Corollary 1 to study the existence of continuous solutions to the nonlinear functional integral equations (2).

Let us now introduce the following assumptions.

(H0)

(i) The function $\sigma: J \longrightarrow J$ is continuous and nondecreasing

(ii) The function $q: J \longrightarrow \mathbb{R}$ is continuous

(iii) The function $a: J \longrightarrow X$ is continuous, and $\|a\|_{\infty}<1$

(H1) The operator $k: J \times X \longrightarrow X$ is such that

(i) For all $t \in J$, the operator $k(t, \cdot): X \longrightarrow X$ is weakly sequentially continuous and weakly compact.

(ii) For each $x \in X$, the operator $k(\cdot, x): J \longrightarrow X$ is continuous.

(iii) There exists a continuous function $\delta: J$ $\longrightarrow[0,+\infty)$ with bound $\Delta=\|\delta\|_{\infty}$ such that 
(H2) The operator $g: J \times J \times X \longrightarrow \mathbb{R}$ is continuous such that, for each $s \in J$ and $x \in X$, and the operator $g(\cdot, s, t): J \longrightarrow \mathbb{R}$ is continuous uniformly.

(H3) The operator $\phi: J \times X \longrightarrow \mathbb{R}$ is such that (i) For each $t \in J$, the operator $\phi(t, \cdot): X \longrightarrow \mathbb{R}$ is weakly sequentially continuous.

(ii) For each $x \in X$, the operator $\phi(\cdot, x): J \longrightarrow \mathbb{R}$ is continuous.

(iii) There exists a constant $L$ such that

$$
|\phi(t, x)-\phi(t, y)| \leq L\|x-y\|, \quad \text { for all } x, y \in X \text { and } t \in J .
$$

(H4) The operatorsw: $J \times X \longrightarrow \mathbb{R}$ is such that

(i) For each $x \in X$, the operator $w(\cdot, x): J \longrightarrow \mathbb{R}$ is measurable.

(ii) For each $t \in J$, the operator $w(t, \cdot): X \longrightarrow \mathbb{R}$ is weakly sequentially continuous.

(iii) There exists a constant $b$ and a function $m \in L^{1}([0,1])$ such that

$$
|w(t, x)| \leq m(t) \text { and } \int_{0}^{1} \frac{1}{t+s} m(s) \mathrm{d} s \leq b .
$$

(H5) Assume that there exists $r_{0}>1$ such that $|g(\cdot, \cdot, \cdot)| \leq r_{0}-\|q\|_{\infty}, \quad \sup _{t \in J}|w(t, 0)| \leq(((1-\| a$ $\left.\left.\left.\|_{\infty}\right) r_{0}-L\|m\|_{L^{1}}\|v\|_{\infty}\right) /\|v\|_{\infty}\right)$, and $\sup _{t \in J}|k(t, 0)|$ $\leq r_{0}\left(1-\Delta-\|u\|_{\infty}\right)$.

Let us define the subset $\Omega$ of $\mathscr{C}(J, X)$ by

$$
\Omega=\left\{x \in \mathscr{C}(J, X):\|x\| \leq r_{0}\right\} .
$$

We can see that $\Omega$ is a nonempty closed and convex subset of $E$. Let $U$ be a weakly open subset of $\Omega$ such that $0 \in \bar{U}$. Notice that (2) is equivalent to the system:

$$
\left\{\begin{array}{l}
x(t)=A x(t)+B y(t), \\
y(t)=C x(t)+D y(t),
\end{array}\right.
$$

where the operators $A, B, C$, and $D$ defined by

$$
\begin{aligned}
& A x(t)=k(t, x(t)), \quad \text { for all } t \in J, \\
& B x(t)=\left[q(t)+\int_{0}^{\sigma(t)} g(t, s, x(s)) \mathrm{d} s\right] \cdot u, \quad \text { for all } t \in J, \\
& C x(t)=\phi\left(t, \int_{0}^{t} \frac{t}{t+s} w(s, x(s)) \mathrm{d} s\right) \cdot v, \quad \text { for all } t \in J, \\
& D y(t)=a(t) y(t), \quad \text { for all } t \in J .
\end{aligned}
$$

Now, we have come to a place where we give the main result of this section.

Theorem 11. Assume that $\left(H_{0}\right),\left(H_{1}\right),\left(H_{2}\right),\left(H_{3}\right),\left(H_{4}\right)$, and $\left(H_{5}\right)$ hold. In addition, assume that

$$
\lambda A u_{1}+\lambda B u_{2} \neq u_{1}
$$

for all $\left(u_{1}, u_{2}\right) \in \partial_{\Omega} U \times X$ and $\lambda \in(0,1)$.

Then, system (2) has, at least, one solution in $\mathscr{C}(J, X) \times \mathscr{C}(J, X)$.

Proof. In order to apply Corollary 1, we divided the proof into four steps.

Step 1: in this step, we prove in (a), (b), and (c), respectively, that the operators $A, B$, and $C$ are well defined and weakly sequentially continuous.

(a) Let $x \in \mathscr{C}(J, X)$, and let $\left\{t_{n}\right\}_{n}$ be a sequence in $J$ such that $t_{n} \longrightarrow t \in J$. We have

$$
\begin{aligned}
\left\|A x\left(t_{n}\right)\left(t_{n}\right)-A x(t)\right\| & =\left\|k\left(t_{n}, x\left(t_{n}\right)\right)-k(t, x(t))\right\| \leq\left\|k\left(t_{n}, x\left(t_{n}\right)\right)-k\left(t_{n}, x(t)\right)\right\|+\left\|k\left(t_{n}, x(t)\right)-k(t, x(t))\right\| \\
& \leq \delta\left(t_{n}\right)\left\|x\left(t_{n}\right)-x(t)\right\|+\left\|k\left(t_{n}, x(t)\right)-k(t, x(t))\right\| \leq \Delta\left\|x\left(t_{n}\right)-x(t)\right\|+\left\|k\left(t_{n}, x(t)\right)-k(t, x(t))\right\| .
\end{aligned}
$$

Since $t_{n} \longrightarrow t$ and taking into account the assumptions $\left(\mathrm{H}_{1}\right)(\mathrm{ii})$, we obtain $\| A x\left(t_{n}\right)$ $-A x(t) \| \longrightarrow 0$. Accordingly, $A x \in E$. Now, we show that $A$ is weakly sequentially continuous. To see this, let $\left\{x_{n}\right\}_{n}$ be a sequence in $\Omega$ such that $x_{n} \rightarrow x \in \Omega$. Hence, $\left\{x_{n}\right\}_{n}$ is bounded; then, by Lemma 3 , we have $x_{n}(t) \rightarrow x(t)$, for all $t \in J$; consequently, 
$A x_{n}(t)=k\left(t, x_{n}(t)\right) \rightarrow k(t, x(t))=A x(t)$.

Using again Lemma 3 , we get $A x_{n} \rightarrow A x$ because $\left\{A x_{n}\right\}_{n}$ is bounded by $\Delta r_{0}+\sup _{t \in J}\|k(t, 0)\|$; hence, $A$ is weakly sequentially continuous. (b) Let $x \in \mathscr{C}(J, X)$, and let $\left\{t_{n}\right\}_{n}$ be a sequence in $J$ such that $t_{n} \longrightarrow t \in J$. We have

$$
\begin{aligned}
\left\|B x\left(t_{n}\right)-B x(t)\right\| & \leq\left|\int_{0}^{\sigma\left(t_{n}\right)} g\left(t_{n}, s, x(s)\right) \mathrm{d} s-\int_{0}^{\sigma(t)} g(t, s, x(s)) \mathrm{d} s\right|\|u\|_{\infty}+\left|q\left(t_{n}\right)-q(t)\right|\|u\|_{\infty} \\
& \leq\left[\int_{0}^{\sigma\left(t_{n}\right)}\left|g\left(t_{n}, s, x(s)\right)-g(t, s, x(s))\right| \mathrm{d} s\right]\|u\|_{\infty}+\left[\left|\int_{\sigma\left(t_{n}\right)}^{\sigma(t)} g(t, s, x(s)) \mathrm{d} s\right|+\left|q\left(t_{n}\right)-q(t)\right|\right]\|u\|_{\infty} \\
& \leq\left[\int_{0}^{1}\left|g\left(t_{n}, s, x(s)\right)-g(t, s, x(s))\right| \mathrm{d} s\right]\|u\|_{\infty}+\left[r_{0}\left|\sigma\left(t_{n}\right)-\sigma(t)\right|+\left|q\left(t_{n}\right)-q(t)\right|\right]\|u\|_{\infty} .
\end{aligned}
$$

By using assumption $\left(\mathrm{H}_{2}\right)$ with the dominated convergence theorem, we obtain

$$
\int_{0}^{1}\left|g\left(t_{n}, s, x(s)\right)-g(t, s, x(s))\right| \mathrm{d} s \longrightarrow 0 .
$$

Hence,

$$
\left\|B x\left(t_{n}\right)-B x(t)\right\| \longrightarrow 0,
$$

This implies that the function $B x$ is continuous. Now, let $\left\{x_{n}\right\}_{n}$ be a sequence in $E$ such that $x_{n} \rightarrow x \in E$. For all $t \in J$, we have $B x_{n}(t)=r_{n}(t) \cdot u$, where

$$
r_{n}(t)=q(t)+\int_{0}^{\sigma(t)} g\left(t, s, x_{n}(s)\right) \mathrm{d} s .
$$

Because $\left\{r_{n}(t), n \in \mathbb{N}\right\}$ is a real bounded sequence, we deduce that there is a renamed subsequence such that $r_{n}(t) \longrightarrow r(t)$. Since $\left\{B x_{n}, n \in \mathbb{N}\right\}$ is bounded, we obtain $B x_{n} \rightarrow B x$; hence, $B$ is weakly sequentially continuous.

(c) Let $x \in \mathscr{C}(J, X)$, and let $\left\{t_{n}\right\}_{n}$ be a sequence in $J$ such that $t_{n} \longrightarrow t \in J$. We have

$$
\begin{aligned}
& \left\|C x\left(t_{n}\right)-C x(t)\right\| \leq\left|\phi\left(t_{n}, \int_{0}^{t_{n}} \frac{t_{n}}{t_{n}+s} w(s, x(s)) \mathrm{d} s\right)-\phi\left(t, \int_{0}^{t} \frac{t}{t+s} w(s, x(s)) \mathrm{d} s\right)\right|\|v\|_{\infty} \\
& \leq\left|\phi\left(t_{n}, \int_{0}^{t_{n}} \frac{t_{n}}{t_{n}+s} w(s, x(s)) \mathrm{d} s\right)-\phi\left(t_{n}, \int_{0}^{t} \frac{t}{t+s} w(s, x(s)) \mathrm{d} s\right)\right|\|v\|_{\infty} \\
& +\left|\phi\left(t_{n}, \int_{0}^{t} \frac{t}{t+s} w(s, x(s)) \mathrm{d} s\right)-\phi\left(t, \int_{0}^{t} \frac{t}{t+s} w(s, x(s)) \mathrm{d} s\right)\right|\|v\|_{\infty} \\
& \leq L\left|\int_{0}^{t_{n}} \frac{t_{n}}{t_{n}+s} w(s, x(s)) \mathrm{d} s-\int_{0}^{t} \frac{t}{t+s} w(s, x(s)) \mathrm{d} s\right|\|v\|_{\infty} \\
& +\left|\phi\left(t_{n}, \int_{0}^{t} \frac{t}{t+s} w(s, x(s)) \mathrm{d} s\right)-\phi\left(t, \int_{0}^{t} \frac{t}{t+s} w(s, x(s)) \mathrm{d} s\right)\right|\|v\|_{\infty} \\
& \leq L\left|\int_{0}^{t_{n}} \frac{s\left(t_{n}-t\right)}{\left(t_{n}+s\right)(t+s)} w(s, x(s)) \mathrm{d} s-\int_{t_{n}}^{t} \frac{t}{t+s} w(s, x(s)) \mathrm{d} s\right|\|v\|_{\infty} \\
& +\left|\phi\left(t_{n}, \int_{0}^{t} \frac{t}{t+s} w(s, x(s)) \mathrm{d} s\right)-\phi\left(t, \int_{0}^{t} \frac{t}{t+s} w(s, x(s)) \mathrm{d} s\right)\right|\|v\|_{\infty} \\
& \leq 2 L b\left|t_{n}-t\right|\|v\|_{\infty}+\left\|\phi\left(t_{n}, z\right)-\phi(t, z)\right\|\|v\|_{\infty}
\end{aligned}
$$


where

$z(t)=\int_{0}^{t} \frac{t}{t+s} w(s, x(s)) \mathrm{d} s, \quad$ for all $t \in J$.

If we refer to assumption $\left(\mathrm{H}_{3}\right)(\mathrm{ii})$, we deduce $C x$ is continuous.

Now, let $\left\{x_{n}\right\}_{n}$ be a sequence in $E$ such that $x_{n} \rightarrow x \in E$; using assumption $\left(\mathrm{H}_{4}\right)$ (ii), we have

$\frac{t}{t+s} w\left(s, x_{n}(s)\right) \rightarrow \frac{t}{t+s} w(s, x(s)), \quad$ in $\mathbb{R}$.

Using the dominated convergence theorem, we obtain

$$
\left(C x_{n}\right)(t) \rightarrow(C x)(t), \quad \text { in } X .
$$

Since $\left\{C x_{n}, n \in \mathbb{N}\right\}$ is bounded, then we can apply Lemma 3 , and we get $C x_{n}-C x$; then, $C$ is weakly sequentially continuous.

Step 2: in this step, in (a) and (b), respectively, we prove that $C\left(\overline{U^{w}}\right)$ and $A\left(\overline{U^{w}}\right)$ are relatively weakly compact.

(a) Let $\left\{x_{n}\right\}_{n}$ be any sequence in $\Omega$. By $\left(\mathrm{H}_{3}\right)$ (iii), we have

$$
\begin{aligned}
\left\|C x_{n}(t)\right\| & =\left[\left|\phi\left(t, \int_{0}^{t} \frac{t}{t+s} w\left(s, x_{n}(s)\right) \mathrm{d} s\right)-\phi(t, 0)\right|+|\phi(t, 0)|\right]\|v\|_{\infty} \\
& \leq\left[L\left|\int_{0}^{t} \frac{t}{t+s} w\left(s, x_{n}(s)\right) \mathrm{d} s\right|+\sup _{t \in J}|w(t, 0)|\right]\|v\|_{\infty} \\
& \leq\left[L\|m\|_{L^{1}}+\sup _{t \in J}|w(t, 0)|\right]\|v\|_{\infty} .
\end{aligned}
$$

For all $t \in[0,1]$, this proves that $\left\{C x_{n}, n \in \mathbb{N}\right\}$ is a uniformly bounded sequence in $C(\Omega)$. As a result, $\left|x^{*}\left(\left(C x_{n}\right)(t)-\left(C x_{n}\right)\left(t^{\prime}\right)\right)\right| \leq[L|M(\varepsilon)|+|\Phi(\varepsilon)|]\left\|x^{*}(v)\right\|$, $C(\Omega)(t)$ is sequentially relatively weakly compact. Now, we proceed to show that it is also weakly equicontinuous. If we take $\varepsilon>0, x \in \Omega$, and $t, t^{\prime} \in[0,1]$ (without loss of generality assume that $\left.t<t^{\prime}\right)$, then we have

$$
\begin{aligned}
& M(\varepsilon)=\int_{0}^{t} \frac{t}{t+s} w\left(s, x_{n}(s)\right) \mathrm{d} s-\int_{0}^{t^{\prime}} \frac{t^{\prime}}{t^{\prime}+s} w\left(s, x_{n}(s)\right) \mathrm{d} s, \quad\left|t-t^{\prime}\right|<\varepsilon, \text { and } \\
& \Phi(\varepsilon)=\phi\left(t, \int_{0}^{t^{\prime}} \frac{t^{\prime}}{t^{\prime}+s} w(s, x(s)) \mathrm{d} s\right)-\phi\left(t^{\prime}, \int_{0}^{t^{\prime}} \frac{t^{\prime}}{t^{\prime}+s} w(s, x(s)) \mathrm{d} s\right), \quad\left|t-t^{\prime}\right|<\varepsilon .
\end{aligned}
$$

Using assumption $\left(\mathrm{H}_{4}\right)$, we have

$$
\begin{aligned}
|M(\varepsilon)| & \leq\left|t-t^{\prime}\right| \int_{0}^{1} \frac{1}{t+s} w\left(s, x_{n}(s)\right) \mathrm{d} s+\int_{t^{\prime}}^{t} \frac{t^{\prime}}{t^{\prime}+s} w\left(s, x_{n}(s)\right) \mathrm{d} s \\
& \leq a\left|t-t^{\prime}\right|+\int_{t^{\prime}}^{t} m(s) \mathrm{d} s .
\end{aligned}
$$

Since $M(\varepsilon) \longrightarrow 0$ and $\Phi(\varepsilon) \longrightarrow 0$, as $\varepsilon \longrightarrow 0$, we obtain

$\left|x^{*}\left(\left(C x_{n}\right)(t)-\left(C x_{n}\right)\left(t^{\prime}\right)\right)\right| \longrightarrow 0, \quad$ as $t \longrightarrow t^{\prime}$.
Applying the Arzelà-Ascoli's theorem [22], we get $C(\Omega)$ is sequentially relatively weakly compact in $X$, and an application of Eberlein-Smulian's theorem [23] shows that $C(\Omega)$ is relatively weakly compact. 
(b) We have $A(\Omega)=\left\{A x:\|x\| \leq r_{0}\right\}$; this subset is nothing else than

$$
k(\cdot, \Omega)=\left\{k(\cdot, x):\|x\| \leq r_{0}\right\},
$$

Hence, by hypothesis $\left(\mathrm{H}_{1}\right)(\mathrm{i})$, we can see that $A(\Omega)$ is relatively weakly compact.

Step 3: in this step in (a), we show that $C\left(\overline{U^{w}}\right) \subset(I-D)(\Omega)$, and in (b), we prove that $A x+B(I-D)^{-1} C x \in \Omega$, for all $x \in \overline{U^{w}}$.

(a) Let $x \in \overline{U^{w}}$, we defined the mapping $\phi_{x}: \mathscr{C}(J, X) \longrightarrow \mathscr{C}(J, X)$ by $\phi_{x}(y)=C x+D y$. Because $D$ is a contraction, we can see that $\phi_{x}$ is a contraction; then, an application of Banach's fixed point theorem yields there is a unique point $y \in \mathscr{C}(J, X)$ such that $y=C x+D y$, and this implies that $C\left(\overline{U^{w}}\right) \subset(I-D)(\mathscr{C}(J, X))$. Since $y \in \mathscr{C}(J, X)$, then there is $t^{*} \in J$ such that

$$
\begin{aligned}
\|y\|_{\infty} & =\left\|y\left(t^{*}\right)\right\| \\
& =C x\left(t^{*}\right)+D y\left(t^{*}\right) \\
& \leq\left|\phi\left(t^{*}, \int_{0}^{t^{*}} \frac{t^{*}}{t^{*}+s} w\left(s, x_{n}(s)\right) \mathrm{d} s\right)\right|\|v\|_{\infty}+\|a\|_{\infty}\left\|y\left(t^{*}\right)\right\| .
\end{aligned}
$$

This implies that

$$
\begin{array}{r}
\|y\|_{\infty} \leq \frac{\left[L\|m\|_{L^{1}}+\sup _{t \in J}|w(t, 0)|\right]\|v\|_{\infty}}{1-\|a\|_{\infty}} \\
\leq r_{0} .
\end{array}
$$

Then, $C\left(\overline{U^{w}}\right) \subset(I-D)(\Omega)$.

(b) Let $y \in \mathscr{C}(J, X)$ and $x \in \overline{U^{w}}$ such that

$$
y(t)=A x(t)+B(I-D)^{-1} C x(t) .
$$

or, equivalently, for all $t \in J$,

$$
y(t)=A x(t)+B(I-D)^{-1} C x(t) .
$$

We have

$$
\begin{aligned}
\|y(t)\| & =\left\|A x(t)+B(I-D)^{-1} C x(t)\right\| \\
& \leq\|A x(t)\|+\left\|B(I-D)^{-1} C x(t)\right\| \\
& \leq \Delta\|x(t)\|+\sup _{t \in J}|k(t, 0)|+r_{0}\|u\|_{\infty} \\
& \leq \Delta r_{0}+\sup _{t \in J}|k(t, 0)|+r_{0}\|u\|_{\infty} \\
& \leq r_{0} .
\end{aligned}
$$

This implies that

$$
A x+B(I-D)^{-1} C x \in \Omega, \quad \text { for all } x \in \overline{U^{w}} .
$$

Thus, all the hypotheses of Corollary 1 are satisfied, and therefore, system (2) has, at least, one solution in $\mathscr{C}(J, X) \times \mathscr{C}(J, X)$.

\section{Example}

Consider the Banach space $E=\mathscr{C}([0,1], \mathbb{R})$ of all continuous real-valued functions on $J=[0,1]$, with norm $\|x\|_{\infty}=\sup _{t \in[0,1]}|x(t)|$. In this case, $X=\mathbb{R}$, and $E$ is a reflexive Banach space. We consider the following coupled nonlinear integral equation in $E$ :

$$
\left\{\begin{array}{l}
x(t)=\frac{1}{4} t^{3} \cos \left(\frac{x(t)}{2}\right)+\frac{1}{8}\left[\frac{1}{4}+\int_{0}^{t} \frac{|y(s)|}{1+|y(s)|} \mathrm{d} s\right], \\
y(t)=t \int_{0}^{t} \frac{t s|x(s)|}{(t+s) e^{|x(s)|}} \mathrm{d} s+\frac{1}{3} \sin (t) y(t) .
\end{array}\right.
$$

To show that (54) has a solution in $E$, we will verify that all conditions of Theorem 11 are satisfied.

Here, for all $t, s \in J$ and $x, y \in E$, we have

$$
\begin{aligned}
\sigma(t) & =t, \\
q(t) & =\frac{1}{4}, \\
a(t) & =\frac{1}{3} \sin (t), \\
u & =\frac{1}{8}, \\
v & =1,
\end{aligned}
$$

$$
\phi(t, x(t))=t x(t)
$$$$
k(t, x(t))=\frac{1}{4} t^{3} \cos \left(\frac{x(t)}{2}\right),
$$

$$
\begin{aligned}
g(t, s, y(s)) & =\frac{|y(s)|}{1+|y(s)|}, \\
w(s, x(s)) & =\frac{s(|x(s)|+1)}{2 e^{|x(s)|+1}} .
\end{aligned}
$$

For each $t \in J$, the operator $k(t, \cdot): X \longrightarrow X$ is continuous (then weakly sequentially continuous), and for each $x \in X$, the operator $k(\cdot, x): J \longrightarrow X$ is continuous. Now, let $x, y \in E$ and $t \in J$, and we have

$$
|k(t, x(t))-k(t, y(t))| \leq \frac{1}{8} t^{3}|x(t)-y(t)|
$$

where the function $\delta: t \longrightarrow 1 / 8 t^{3}$ is continuous with bound $\Delta=1 / 8$.

Next, we have $g$ is continuous, and for each $s \in J$ and $x \in \mathbb{R}$, the operator $g(\cdot, s, t): J \longrightarrow \mathbb{R}$ is continuous uniformly.

Moreover, for all $x, y \in \mathbb{R}$ and $t \in J$, we have 


$$
|\phi(t, x(t))-\phi(t, y(t))| \leq|x(t)-y(t)| .
$$

Thus, $L=1$.

Next, we have

$$
\begin{aligned}
|w(t, x(t))| & \leq \frac{t}{2}, \\
\text { and } \int_{0}^{1} \frac{1}{t+s} m(s) \mathrm{d} s & \leq 1 .
\end{aligned}
$$

where $m(t)=t$, for all $t \in J$.

Choose $b=1$ and $r_{0}=2$, we obtain $r_{0}-\|q\|_{\infty}=7 / 4$, $\left(\left(\left(1-\|a\|_{\infty}\right) r_{0}-L\|m\|_{L^{1}}\|v\|_{\infty}\right) /\|v\|_{\infty}\right)=5 / 6, \quad$ and $r_{0}\left(1-\Delta-\|u\|_{\infty}\right)=3 / 2$. Then, the inequalities $|g(\cdot, \cdot, \cdot)| \leq 7 / 4, \sup _{t \in J}|w(t, 0)| \leq 5 / 6$, and $\sup _{t \in J}|k(t, 0)| \leq 3 / 2$ are verified.

Now, let $U=\{x \in \mathscr{C}([0,1], \mathbb{R}):\|x\|<2\}, \quad$ we have $\partial U=\{x \in \mathscr{C}([0,1], \mathbb{R}):\|x\|=2\}$. Suppose that there exists $u_{1} \times u_{2} \in \partial U \times X$ and $\lambda \in(0,1)$ such that $\lambda A u_{1}+\lambda B u_{2}=u_{1}$. We have $\left\|\lambda A u_{1}+\lambda B u_{2}\right\|<13 / 32$. And, $\left\|u_{1}\right\|=2$ which is a contraction. Hence, for all $u_{1} \times u_{2} \in \partial U \times X$ and $\lambda \in(0,1)$, we have $\lambda A u_{1}+\lambda B u_{2} \neq u_{1}$. Hence, all conditions of Theorem 11 are verified.

\section{Conclusion}

In recent years, some works were devoted to the investigation of fixed point theorems for operator matrices with entries acting on Banach spaces and Banach algebras. The aim of the present paper was to establish some new variants of Leray-Schauder-type fixed point theorems for a $2 \times 2$ block operator matrix. The second aim of this study was to use our results to prove the existence of solutions for a coupled system of nonlinear equations. An example to illustrate our theory is included.

\section{Data Availability}

No data were used to support this study.

\section{Conflicts of Interest}

The authors declare that they have no conflicts of interest.

\section{References}

[1] A. Jeribi, B. Krichen, and B. Mefteh, "Existence of solutions of a two-dimensional boundary value problem for a system of nonlinear equations arising in growing cell populations," Journal of Biological Dynamics, vol. 7, no. 1, pp. 218-232, 2013.

[2] N. Kaddachi, A. Jeribi, and B. Krichen, "Fixed point theorems of block operator matrices on banach algebras and an application to functional integral equations," Mathematical Methods in the Applied Sciences, vol. 36, no. 6, pp. 659-673, 2013.

[3] A. Jeribi, N. Kaddachi, N. Kaddachi, and B. Krichen, "Existence results for a system of nonlinear integral equations in Banach algebras under weak topology," Fixed Point Theory, vol. 18, no. 1, pp. 247-268, 2017.

[4] A. Jeribi and B. Krichen, "Nonlinear functional analysis in banach spaces and banach algebras, fixed point theory under weak topology for nonlinear operators and block operator matrices with applications," in Monographs and Research Notes in Mathematics, CRC Press, New York, NY, USA, 2015.

[5] N. Kaddachi, "Generalized form of fixed point theorems in Banach algebras under weak topology with an application," Filomat, vol. 33, no. 13, pp. 4281-4296, 2019.

[6] A. Amar, A. Jeribi, and B. Krichen, "Fixed point theorems for block operator matrix and an application to a structured problem under boundary conditions of Rotenberg's model type," Mathematica Slovaca, vol. 64, no. 1, pp. 155-174, 2014.

[7] M. Rotenberg, "Transport theory for growing cell populations," Journal of Theoretical Biology, vol. 103, no. 2, pp. 181-199, 1983.

[8] A. Jeribi, N. Kaddachi, and B. Krichen, "Fixed-point theorems for multivalued operator matrix under weak topology with an application," Bulletin of the Malaysian Mathematical Sciences Society, vol. 43, no. 2, pp. 1047-1067, 2020.

[9] De Blasi and S. Francesco, "On a property of the unit sphere in a Banach space," Bulletin Mathematique de la Societe des Sciences Mathematiques de Roumanie, vol. 21, pp. 259-262, 1977.

[10] J. Appell and E. De Pascale, "Su alcuni parametri connessi con la misura di non compattezza di Hausdorff in spazi di funzioni misurabili," Bollettino dell'Unione Matematica Italiana, vol. 6, no. 3-B, pp. 497-515, 1984.

[11] Y. Liu and Z. Li, "Schaefer type theorem and periodic solutions of evolution equations," Journal of Mathematical Analysis and Applications, vol. 316, no. 1, pp. 237-255, 2006.

[12] D. W. Boyd and J. S. W. Wong, "On nonlinear contractions," Proceedings of the American Mathematical Society, vol. 20, no. 2, p. 458, 1969.

[13] T. Xiang and R. Yuan, "Critical type of Krasnosel'skii fixed point theorem," Proceedings of the American Mathematical Society, vol. 139, no. 3, Article ID 1033, 2011.

[14] A. B. Amar and M. Mnif, "Leray-Schauder alternatives for weakly sequentially continuous mappings and application to transport equation," Mathematical Methods in the Applied Sciences, vol. 33, no. 1, pp. 80-90, 2010.

[15] A. B. Amar, S. Derbel, D. O’Regan, and T. Xiang, "Fixed point theory for countably weakly condensing maps and multimaps in non-separable Banach spaces," Journal of Fixed Point Theory and Applications, vol. 21, no. 1, p. 8, 2019.

[16] R. P. Agarwal, D. O’Regan, and M. A. Taoudi, "Browder Krasnoselskii-type fixed point theorems in Banach spaces," Fixed Point Theory and Applications, vol. 2010, no. 1, Article ID 243716, 2010.

[17] I. Dobrakov, "On representation of linear operators on $C_{0}(T$, X)," Czechoslovak Mathematical Journal, vol. 21, no. 1, pp. 13-30, 1971.

[18] H. Brezis, "Analyse fonctionnelle," Théorie et Applications, Masson, Paris, France, 1983.

[19] A. Ben Amar, I. Feki, and A. Jeribi, "Leray-Schauder and FuriPera types fixed point theorems for the sum of two weakly sequentially continuous mappings and application to transport equation," Afrika Matematika, vol. 25, no. 3, pp. 707-722, 2014.

[20] A. Khchine, L. Maniar, and M.-A. Taoudi, "Leray-Schaudertype fixed point theorems in Banach algebras and application to quadratic integral equations," Fixed Point Theory and Applications, vol. 2016, no. 1, p. 88, 2016.

[21] T. Xiang and S. G. Georgiev, "Noncompact-type Krasnoselskii fixed-point theorems and their applications," Mathematical Methods in the Applied Sciences, vol. 39, no. 4, pp. 833-863, 2016.

[22] I. I. Vrabie, A. I. Cuza, and O. Mayer, $C_{0}-$ Semigroups and Applications, Elsevier, New-York, NY, USA, 2003.

[23] J. B. Conway, A Course in Functional Analysis, Springer, Berlin, Germany, 2nd edition, 1990. 